\title{
Derivation of a Reliability Metric for Fused Data Decision Making
}

\author{
Erik P. Blasch \\ Air Force Research Lab \\ 2241 Avionics Cir \\ WPAFB, OH 45433 \\ erik.blasch@,wpafb.af.mil
}

\begin{abstract}
Users require information fusion to reduce dimensionality for real world, complex decision-making. Typically, researchers design fusion systems based on limited data that does not capture all operating conditions seen in the real world (i.e. weather for video sensors). Fusion systems are of limited use when presented with poor data, inappropriate models, and unrealistic assumptions. Decision makers are burdened with the task of determining the quality of fused output based on trial and error. If the fusion system works in most scenarios, aids the user in purposeful decisions, and leads to successful actions; the user has high system confidence. However, if the fusion system causes erroneous results that lead to poor decisions, the user disposes of the entire system - which could have been the result of bad data, incomplete models, and restricted assumptions. Thus, when a fusion system is fielded, there has to be metrics associated with model fidelity, data uncertainty, and constraints over applicability. By combing representations of data quality; this paper derives a reliability metric to aid users to trust fusion outputs, perform a utility assessment, and refine sensor collections.
\end{abstract}

Keywords: Metrics, fusion, quality, reliability, fidelity

\section{Introduction}

In 1990, the US Department of Defense defined data fusion as "a technology which involves the acquisition, integration, filtering, correlation and synthesis of useful data from diverse sources for the purposes of situation/environment assessment, planning, detecting, verifying, diagnosing problems, aiding tactical and strategic decisions, and improving systems performance and utility". [1] The definition requires two parts, the machine and the user. The machine does the integration and filtering of "useful data", while the user provides quantitative and qualitative situation assessments and sensor management strategies based on a performance and utility analysis. The unidentified word in the definition is "useful". We propose a reliability metric to quantitatively explain "useful".

The User Fusion model, [2] shown in Figure 1, incorporate six levels of information. Level 0 is the a priori models of the situation of interest. Level 1 is object assessment. Level 2 and 3 are situation assessment and threat assessment. Level 4 is process refinement (or sensor management [3]) and level 5 is User refinement. User refinement requires reliable results for effective decision making. The user evaluates the utility of information based on information needs, cost of action estimates, and data reliability. Reliability requires accurate, confident and timely results. Specifically, for a valid spatiotemporal analysis, reliability requires accurate, confident, and timely results.

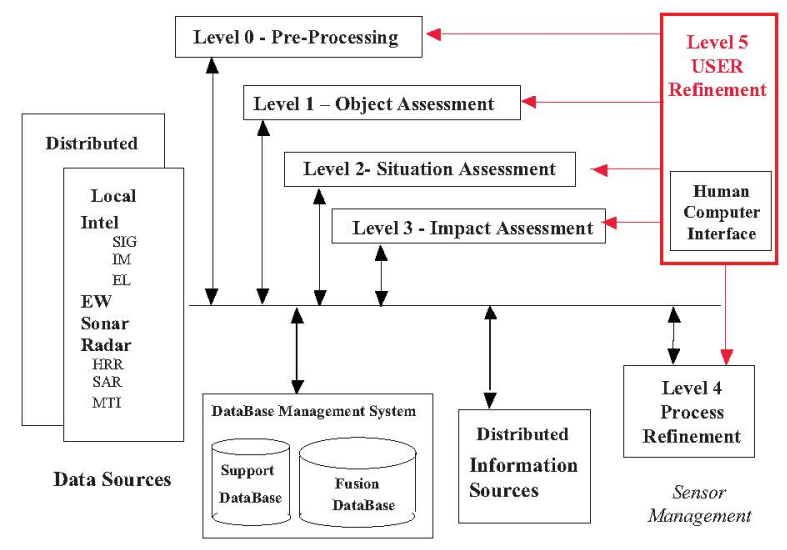

Figure 1. User-Fusion Model

Since users can refine, approve, or negate machinefusion decisions, it is important to know what criteria they used in their decision making process. Many times, the user is forced to combine the presented data, infer unknowns, and decide based on limited or uncertain data. [4] Users typically do not know the quality of the data that is being presented to them, nor do they know when it was collected. In order to establish mission [5] criteria and metrics for decision making, we derive a reliability metric for data assessment. Reliability [6] in the decision making is similar to utility estimation $[7,8,9]$ in that the trust the user has is related to the quality of the data (timeliness, accuracy, and confidence as well as reducability and presentation).

Human computer interface (HCI) approaches emphasize the valuative nature of decision making. Economic decision making models represent values in terms of both utility (or value) functions and relative 
importance weights (or tradeoffs) to distinguish competing evaluation criteria. [10] Such an example is subjective evaluation utility (SEU) theory, which allows user to make decisions. Human factors, medical diagnosis, and information fusion have adopted the model. SEU makes four basic assumptions about decision makers:

(a) They have a clearly defined utility function which allows them to assign a preference for outcomes.

(b) They possess a clear and exhaustive view of the possible alternative strategies available to them.

(c) They create a consistent joint probability distribution of future scenarios associated with each strategy.

(d) They will choose strategies in order to maximize their subjective expected utility.

SEU assumes that decision makers have an undisturbed view of all possible scenarios of action. The formal theory requires that the decision maker comprehend an entire range of possible alternatives, both now and in the future. In actuality, human beings do not work out detailed future scenarios, each complete with conditional probability distributions. Rather, the decision maker is likely to contemplate only a few of the available alternatives. Moreover, there is a wealth of evidence to show that when people consider action options, they often neglect seemingly obvious candidates. In addition, they are relatively insensitive to the number and importance of these omitted alternatives. [11]

SEU assumes a well-defined set of subjective values that are consistent across all aspects of the world. Subjective utilities vary from one type of decision to the next and users will vary their strategies that appear inconsistent. human decision making is severely constrained by its 'keyhole' view of the problem space, or what Simon [12] has dubbed bounded rationality:

The capacity of the human mind for formulating and solving complex problems is very small compared with the size of the problems whose solution is required for objectively rational behavior in the real world - or even for a reasonable approximation of such objective rationality.

This fundamental limitation upon human information processing gives rise to satisficing behavior - the tendency to settle for satisfactory rather than optimal courses of action. This is true both for individual and for collective decision making. SEU has also been addressed by engineers.

Blackman [13] has outlined a utility theory approach to sensor selection for target tracking. Individual sensors are allocated to target tracks so as to maximize system utility. The expected gain in marginal utility associated with the allocation of a particular sensor-to-target track is expressed as the improvement in the kinematic estimate (e.g., the target's range) given a sensor measurement. Kinematic utility is estimated using the prediction covariance matrix of a Kalman filter. While utility theory has attributes for user refinement, it is still subject to data and information reliability constraints. If the data is bad, humans make errors:

Error will be taken as a generic term to encompass all those occasions in which a planned sequence of actions fails to achieve its intended outcome, and when these failures cannot be attributed to the intervention of some chance agency. [14]

Errors were attributed either to irrationality or to unawareness on the part of the perceiver. Thus, human beings are assumed to make decisions according to SEU Theory, to draw inferences from evidence in accordance with logical Bayes principles and to make uncertain judgments.

As Hall states, "the utility of a fusion system must be measured by the extent to which the system supports the intended decision process." [15] A fusion system must reduce uncertainty by increasing the reliability of the data.

This paper proposes a reliability metric based on confidence, accuracy, and timeliness. Section 2 details the user-fusion interaction with resource reliability $\left(\mathrm{R}^{2}\right)$ and user utility $\left(\mathrm{U}^{2}\right)$. Section 3 derives the reliability metric. Section 4 shows a specific example and Section 5 draws conclusions.

\section{User-Fusion Interaction}

The user-fusion interaction is the processing, interpretation and use of data from multiple sources. Both the user and the machine have capabilities and limitations. The human is attention limited, but can reason over reliable data. The fusion system can process large quantities of data, reduce dimensionality, and provide data uncertainty estimates to provide reliable information to the user. With reliable estimates, the user can reason over the utility of actions for sensor management and data collections. Figure 2 illustrates the relationship between the user and the machine. The human expects reliable data to perform a utility analysis and sensor allocation refinement.

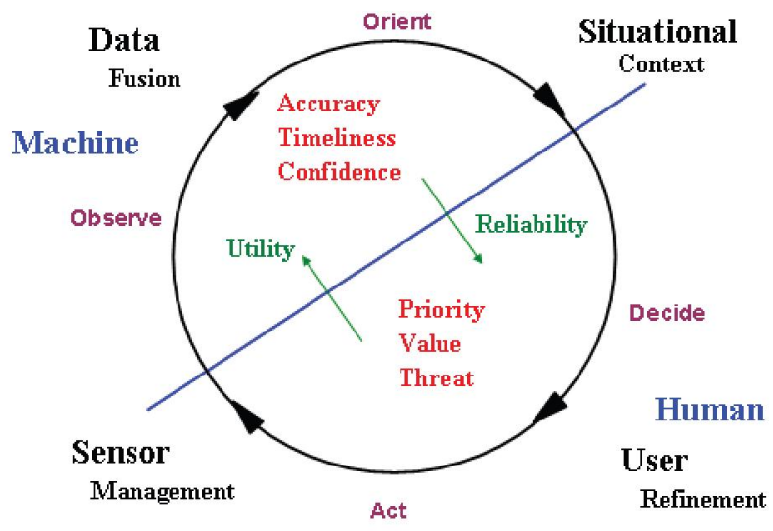

Figure 2. Human-Machine Interaction. 
The usability of fusion systems is based on increased capabilities to process large quantities of data with no loss of functionality or fidelity. The fusion system must use high fidelity models that are appropriate to meet information needs. Low fidelity models ignore potentially important features of the problem whereas too much fidelity makes the system hard to use. The "right" level of fidelity may vary from one user to another. Functionality means easy to use, which includes parsimonious information that is timely, accurate, and confident presented to the user in an intuitive manner. If the fusion system is difficult to use, it probably won't be. These relationships are shown in Figure 3.

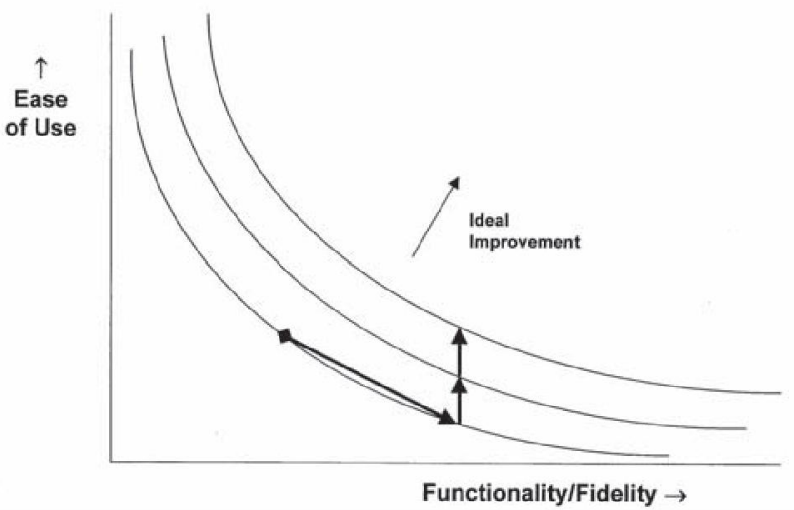

Figure 3. Ease of Use versus functionality

Intent can be a game-theoretic analysis of an adversary. Finally, the value associated with the event or object of interest weight the importance of the attribute measured.

\subsection{Machine-Fusion Reliability}

Data and information reliability implies minimum uncertainty. Uncertainty can be described in many ways such as signals (i.e. noise and bias) and exploitation (i.e. confidence). While noise is inherent to the sensor, bias can result through slowly varying parameters. To compensate for bias, robust control can be used; however, sensor noise is always present. To address data reliability, the fusion system performance metrics are timeliness, accuracy, and confidence. For example, in a track and ID system, we have the expected time (T), accuracy (A), and target ID confidence (C). A system reliability (R) metric, scaled from 0 to 1 , is a multiple of probabilities.

$$
\mathrm{R}_{\mathrm{k}}=\mathrm{A}_{\mathrm{k}} * \mathrm{C}_{\mathrm{k}} * \mathrm{~T}_{\mathrm{k}}
$$

For timeliness and accuracy, we perform a spatiotemporal association of the expected prediction (or sensor quality). If the measurement is immediate and high accurate, then the values are 1 . The reliability metric is a scaled value.

For the analysis, we need to develop a model of confidence, (1 - uncertainty). Many methods exists to create a belief structure (Bayes, Dempster Shafer, NN, etc) to represent confidence. A reliability of 1 is immediate results with zero error and perfect confidence, as shown in Figure 4. In the real world, we have some uncertainty in the position, delay time, and quality of the observation.

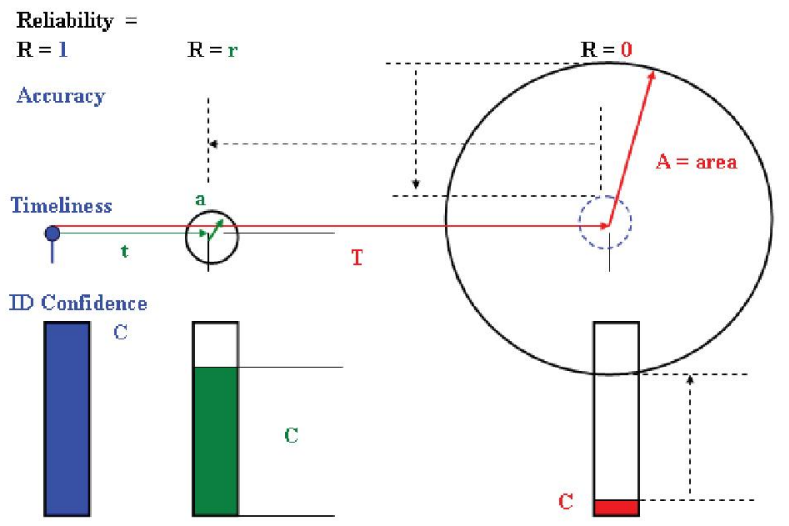

Figure 4. Accuracy, Confidence, and Timing relationship.

In section 3, we seek to combine the quality of service information into a combined reliability metric for variance assessment. First, we must assess the utility of reliable information.

\subsection{User Utility}

Judgment and decision making researchers [16] use utility theory to evaluate possible outcomes, (forwardlooking task). To combine the forward-looking user need for reliable action, we can utilize the fusion system for backward-chaining information. The interaction between the forward and backward data reliability data can ensure that the human has the opportunity for effective decision making. The utility a user places on a system is based on constraints. These constraints can take on many forms such as: intent, value, and ease of use. Since this is a utility metric, we can define the multi-attribute utility (MAU) [1] metric as

$$
\mathrm{U}_{\mathrm{k}}=\mathrm{I}_{\mathrm{k}} * \mathrm{~V}_{\mathrm{k}} * \mathrm{E}_{\mathrm{k}}
$$

where $\mathrm{I}$ is the intent of the object, $\mathrm{V}$ is the value, and $\mathrm{E}$ is the ease of use.

If we desire the user to have a subjective weighted affect on the fusion system to input their desires, then the resulting system is :

$$
\mathrm{R} * \mathrm{U}=\mathrm{ACT}_{\mathrm{k}} * \mathrm{IVE}_{\mathrm{k}}
$$

or for expected utility RE $=$ ACTIVE. We can model this in state-space as: 


$$
\left[\begin{array}{c}
A_{k+1} \\
C_{k+1} \\
T_{k+1}
\end{array}\right]=\left[\begin{array}{lll}
p_{i a} & p_{i c} & p_{i t} \\
p_{v a} & p_{v c} & p_{v t} \\
p_{e a} & p_{e c} & p_{e t}
\end{array}\right]\left[\begin{array}{c}
A_{k} \\
C_{k} \\
T_{k}
\end{array}\right]+\left[\begin{array}{c}
v_{k} \\
w_{k} \\
z_{k}
\end{array}\right]
$$

where the sum of the columns add to 1 . Hence the user can weight the reliability of information based on the utility desired. If the user wants high accuracy, they must wait an infinite amount of time and get high confidence. Likewise, immediate results incurs low accuracy or confidence. One way to model the utility-reliability tradeoff is to perform an assignment analysis.

\subsection{Assignment}

Typical performance measures [1] for sensor-target paring have been expressed in an assignment matrix. Using the probability matrix $\mathbf{P}$, the set of weight decisions, $d_{i j}$, where $i, j=1,2, \ldots n$ of integers defines all possible means by which the matrix elements may be chosen to select utility-reliability parings. The overall objective function or measure of performance, $\mathrm{M}$, for any set of $n$ pairs is

$$
\mathbf{M}=\mathbf{P}_{\mathrm{UM}} \cdot \sum_{\mathrm{i} ; \mathrm{j}=1}^{\mathrm{n}} d_{\mathrm{ij}}=\mathrm{P}_{\mathrm{UR}} \cdot D_{\mathrm{IJ}}
$$

subject to $\sum_{\mathrm{i}}=1$ and $\sum_{\mathrm{j}}=1$ constraints.

The permutations for the $3 \times 3$ example may be exhaustively computed as: $(a, i=1 ; c, v=2 ; t, e=3)$

$$
\begin{array}{ll}
\text {MOP}_{1}=d_{11}+d_{22}+d_{33} & \text { MOP }_{4}=d_{12}+d_{23}+d_{31} \\
\text {MOP}_{2}=d_{11}+d_{23}+d_{32} & \text { MOP }_{5}=d_{13}+d_{21}+d_{32} \\
\text {MOP}_{3}=d_{12}+d_{21}+d_{33} & \text { MOP }_{6}=d_{13}+d_{22}+d_{31}
\end{array}
$$

Nash [17] has formulated the general assignment approach that considers the use of multiple sensors against individual targets as well as the constraints of sensor capacity and required target coverage. For our general case, we use to the reliability-utility matrix to perform the assignment. The objective function for assignment is a set of parametric values that provide measures of cost that can be combined (in a weighted product) to form a single objective function to be optimized for assignment. Blackman [13] has described the use of marginal utility as the assignment objective function. Utility is defined as the ratio of current performance (i.e. tracking accuracy, measured as estimation error standard deviation) to desired performance. The desired performance is a standard criteria established by the data fusion system. Marginal utility is defined as the difference between current utility and the expected utility that would be achieved if the metric is allocated to the utility function. Expected utility requires a model of sensor performance to predict sensor reliability for assignment to each candidate utility function. For a simple example: if $\mathrm{p}_{\mathrm{ia}}=\mathrm{p}_{\mathrm{vc}}=\mathrm{p}_{\mathrm{et}}=1$, then

$$
\mathrm{M}=\mathrm{p}_{\mathrm{ia}} \mathrm{A}+\mathrm{p}_{\mathrm{vc}} \mathrm{C}+\mathrm{p}_{\text {et }} \mathrm{T}
$$

which matches where (A), what (C), and when(T) with the appropriate considerations of who (I), how much (V), and how $(\mathrm{E})$, respectively.

\subsection{Multiple Attribute Reliability-Utility}

To evaluate the "best" alternative, multiple measures of merit and multiple conditions can provide very complex combinations of results. Measures of performance and a single effectiveness measure (MOE) are defined for evaluating the alternatives. [1]

Methods of decision analysis have been applied to such problems to evaluate quantitatively among candidates to determine their worth we use a Multiple attribute reliability-utility (MARU) analysis. This technique, based on MAU, develops a hierarchical structure that allows the decision maker to quantify the effectiveness of each factor in the evaluation.

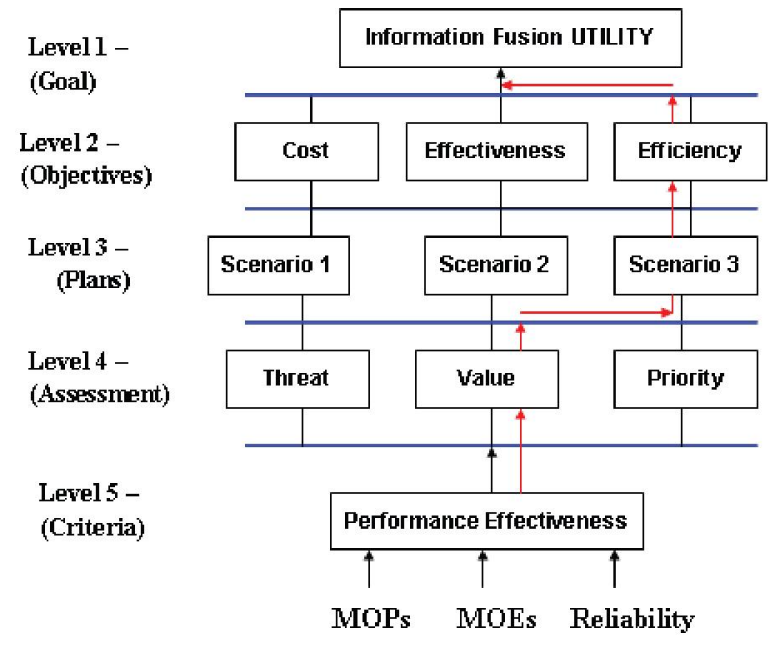

Figure 5. Structure of MAU.

User utility is decomposed into a hierarchy to provide a structure that evaluates measures of merit. Figure 5 depicts the MARU hierarchy. The levels in the figure are

- Level 1 represents the single goal parameter that measures total system worth.

- Level 2 corresponds to three evaluation objectives to be used: cost, effectiveness, and efficiency.

- Level 3 represents the scenarios under which each candidate plan must be evaluated.

- Level 4 represents the utility assessment for a given environmental context.

- Level 5 represents the individual measures of merit provided by the simulation of the RU matrix and the associated reliability of the data.

The arrows depict one path through the hierarchy by which a measure of merit can be related to total worth. Conditioning variables (e. g. scenario contexts) over alternatives and the measurable variables (MOPs, MOEs, MORs) are based on measured and estimated functions for 
U-R probabilities.

The decision-making process includes weighted U-R conditions that contribute to overall value based on user utility and resource reliability. The propagation of measures of merit, upward through utility functions and weights, provides a single quality value for decision making. When properly structured, the resulting scores can be ranked to compare the alternatives.

\subsection{Decision Rules}

In decision making, there are many ways to evaluate the alternatives based on reliability or utility. Expected Multiattribute reliability-utility theory basically weight the desired outcomes for decision making. If $R$ is the data reliability, $U$ the utility desired, and $\mathrm{m}$ is the metric, then we can use these decision rules [15]

1. Maximum a posteriori: Accepts the hypothesis $R_{0}$ as being true if the probability of $R_{0}$ given observation $m$, $\left[\mathrm{P}\left(R_{0} \mid m\right)\right]$, is greater than the probability of $R_{1}$ given $\left[\mathrm{P}\left(R_{1} \mid m\right)\right]$.

2. Maximum Likelihood: Accepts hypothesis $R_{0}$ as true if the a priori probability $R_{0}\left[\mathrm{P}\left(R_{0} \mid m\right)\right]$, multiplied by $\mathrm{P}(m$ $\left.\mid R_{0}\right)$ is greater than $\mathrm{P}\left(R_{1}\right) \cdot \mathrm{P}\left(m \mid R_{1}\right)$.

3. Neyman- Pearson: Accepts the hypothesis $R_{0}$ if the ratio of the likelihood function for $R_{0}$ to the likelihood function for $R_{1}$ is less than or equal to a constant $C$. The constant $C$ is chosen to give the desired significance level.

4. Minimax: A utility (cost) function is established that quantifies the gain (risk) associated with choosing a hypothesis or its alternative. (i.e. choosing the correct sensor-target assignment). The minimax approach selects $R_{0}$ such that the maximizes the utility function or minimizes the cost function.

5. Bayes: A cost function $(C)$ is established that provides a measure of the consequences of choosing hypothesis $R_{0}$ versus $R_{1}$. A typical cost function is given by

$$
\mathrm{C}=\mathrm{C}_{00} \mathrm{P}\left(R_{0}\right) \mathrm{P}_{\mathrm{a}}+\mathrm{C}_{01} \mathrm{P}\left(R_{0}\right) \mathrm{P}_{\mathrm{b}}+\mathrm{C}_{10} \mathrm{P}\left(R_{1}\right) \mathrm{P}_{\mathrm{c}}+\mathrm{C}_{01} \mathrm{P}\left(R_{1}\right) \mathrm{P}_{\mathrm{d}}
$$

where $\mathrm{P}\left(R_{0}\right)$ and $\mathrm{P}\left(R_{1}\right)$ are the a priori probabilities of hypotheses $R_{0}$ and $R_{1}$, respectively. $\mathrm{P}_{\mathrm{a}}$ through $\mathrm{P}_{\mathrm{d}}$ correspond to the probability of a sensor assignment under four possible conditions:

$$
\begin{array}{ll}
\mathrm{P}_{\mathrm{a}}=\mathrm{P}\left\{R_{0}=1 \mid m=1\right\} & \mathrm{P}_{\mathrm{b}}=\mathrm{P}\left\{R_{0}=0 \mid m=1\right\} \\
\mathrm{P}_{\mathrm{c}}=\mathrm{P}\left\{R_{1}=1 \mid m=0\right\} & \mathrm{P}_{\mathrm{d}}=\mathrm{P}\left\{R_{1}=1 \mid m=0\right\}
\end{array}
$$

Hence, $P_{a}$ and $P_{c}$ are detection probabilities, while $P_{b}$ and $P_{d}$ represent the probability of false alarms. The constants $C_{\mathrm{ij}}$ are arbitrarily chosen constants. The Bayes' decision criteria selects $R_{0}$ over $R_{1}$ to minimize the cost function $\mathrm{C}$ or maximize the gain function. [15].

Minimax approaches may use complex cost or utility functions. These functions attempt to develop measures of the cost (liability) or benefit (utility) of choosing one hypotheses over another. For a summary of utility functions for SDT, see Fishburn [18]. As many of these utility approaches are powerful, they can only be appropriate if the data is reliable. We have combined reliability and utility in a fusion scenario based on user refinement strategies and we next show how to model the reliability uncertainty.

\section{Reliability Modeling}

In many fusion systems, we are interested in modeling the spatiotemporal aspects of the system. The spatialtemporal component is a continuous variable while the confidence can be of discrete type. The system reliability can be a fusion of the timeliness, spatial and temporal accuracy, and information confidence. For the accuracy and confidence we use the belief filter [19] approach as was demonstrated for simultaneous tracking and identification. For the information timeliness, we model the usefulness of the information as the inverse of the failure possibilities. For the information confidence, we use an information theoretic analysis. Combining the three probabilities in this first cut to the model, can be a metric of data reliability for decision making. In the next sections, we describe the confidence as a binomial system, a timeliness metric based on the exponential, and the accuracy as a Gaussian system.

\subsection{Accuracy}

Statistical phenomena $[20]$ can be presented by a probability distribution, $P(x)$, and a probability density function $p(x)$.

$$
P(x)=\int_{-\infty}^{x} p(x) d x
$$

The benefit of using a probability model is that many systems are effectively described in terms of only two parameters - a mean $\mu$ and a variance, $(\sigma)^{1 / 2}$. For communication reasons, being able to model the target or clutter with only two parameters is highly desirable.

To model the noise, we use a Gaussian model, where the mean noise is 0 , (i.e. no bias) :

$$
\mathrm{p}(\mathrm{x})=\frac{1}{\sqrt{2 \pi}} \sigma \exp \left(-\frac{\mathrm{x}^{2}}{2 \sigma^{2}}\right)
$$

For the accuracy model, we assess the spatial covariance information. We can use the belief filter which is able to model a confidence (Dempster-Shafer) function with a Bayesian belief in a method akin to Kalman filtering. Assuming a normal distribution over the system, we can assess the covariance matrix as a accuracy reliability. 


\subsection{Timeliness Models}

There are many cases is which a timeliness analysis is completed to determine the operation of a system. Timeliness is associated with usefulness. The assumption is that at some point the data will not be useful and the human will make an incorrect decision (failure or decision-error analysis). In actuality, the human will not an incorrect decision, but an ignorant decision over the information available to them.

We shall use the term system to identify the fusion process and user in combination (FUS) which is to make a decision over some function. We are interested in the cases in which the user is an active participant (INTERACTION). We call the time interval from the moment the system is in operation until it fails (where the user makes an incorrect decision) the time to failure. [Note: if a user makes a correct decision, he succeeds. If the user makes no decision, then either the fusion system makes the decision (monitoring) or the user is passively taking no action]. However, it is assumed that the user must approve or disprove sensor schedules. This interval is, in general, is random, which can be modeled as a random variable $\mathbf{x} \geq 0$. The distribution $F(t)=P\{x \leq t\}$ of this random variable is the probability that the system falls prior to time $t$ where we assume that $t=0$ is the moment the system is starts to received data The difference

$$
R(t)=1-F(t)=\mathrm{P}\{\mathbf{x}>\mathrm{t}\}
$$

is the system timeliness reliability. It equals the probability that the system functions at time $t$. To assess the reliability timeliness, we must assess the point at which the data is not useful.

The mean time or reliability timeliness of a system is the mean of $x$. Since $F(x)=0$ for $x<0$, we conclude that

$$
E\{x\}=\int_{0}^{\infty} x f(x) d x=\int_{0}^{\infty} R(t) d t
$$

The probability that a system functioning at time $t$ falls prior to time $\mathrm{x}>t$ equals

$$
F(x \mid x>t)=\frac{P\{x \leq x ; x>t\}}{P\{x>t\}}=\frac{F(x)-F(t)}{1-F(t)}
$$

Differentiating with respect to $x$, we obtain

$$
f(x \mid x>t)=\frac{f(x)}{1-F(t)}
$$

The product $f(x \mid \mathbf{x}>\mathrm{t}) \mathrm{d} x$ equals the probability that the system falls in the interval $(x, x+d x)$, assuming that it functions at time $t$.

Human decision making (Fitt's Law [21]) develops a time interval over an exponential / lognormal distribution.
In such a case for timelines, we are interested in associating the quality of the data with an exponential distribution in which data that is received quicker is assumed more useful to the user (hence, making correct decisions over the interval from which action is required). If we let the timeliness of the information be associated with some time constant and the appropriate proportionality $f(x)=c e^{-\mathrm{cx}}$, then $F(t)=1-e^{-\mathrm{ct}}$ and

$$
f(x \mid x>t)=\frac{c e^{-c x}}{e^{-c t}}=f(x-t)
$$

This shows that the probability that a system functioning at time $t$ fails in the interval $(x, x+d x)$ depends only on the difference $x-t$ as shown in Figure 6.

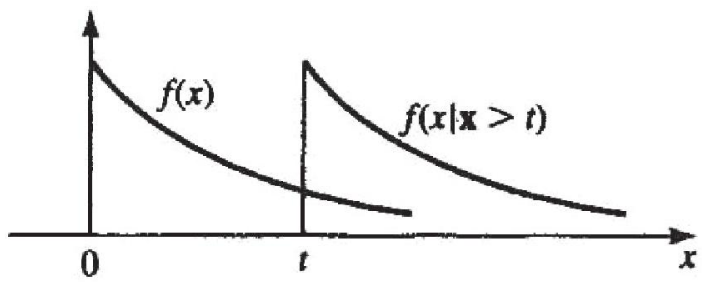

Figure 6. Reliability Timeliness Window.

Conditional Timeliness Rate. The conditional density $f(x$ $\mid \mathbf{x}>\mathrm{t}$ ) is a function of $x$ and $t$. Its value at $\mathrm{x}=t$ is a function only of $t$. This function is denoted by $\beta(\mathrm{t})$ and is called the conditional timeliness rate of the system.

$$
\beta(t)=f(x \mid x>t)=\frac{f(t)}{1-F(t)}
$$

The product $\beta(\mathrm{t}) d t$ is the probability that a system functioning at time $t$ fails in the interval $(t, t+\mathrm{d} t)$. We interpret the function $\beta(t)$ as the expected timeliness rate. From above

$$
\beta(\mathrm{t})=\frac{c e^{-\mathrm{ct}}}{1-\left(1-\mathrm{e}^{-\mathrm{ct}}\right)}=c
$$

It follows that

$$
\beta(t)=\frac{F^{\prime}(t)}{1-F(t)}=-\frac{R^{\prime}(t)}{R(t)}
$$

We shall use this relationship to express the distribution of $\mathbf{x}$ in terms of the function $\beta(t)$. Integrating from 0 to $x$ and using the fact that in $\mathrm{R}(0)=0$, we obtain

$$
-\int_{0}^{\pi} \beta(t) d t=\operatorname{Ln} R(x)
$$

Hence $R(x)=1-F(x)=\exp \left\{-\int_{0}^{x} \beta(t) d t\right\}$ 
And since $f(x)=F^{\prime}(x)$, this yields

$$
f(x)=\beta(x) \exp \left\{-\int_{0}^{x} \beta(t) d t\right\}
$$

\subsection{Information Content}

The information content is modeled as a Binomial trail with the probability being the measured value from a classifier. We use a Bernoulli trail where the information content (or value) is related to the confidence in the reported information. The inverse represents the uncertainty, or unsuredness of the information content.

\subsection{Reliability Assessment}

For the ACT reliability above, we have [20]

$\begin{array}{clcc}\text { Variable } & \mathbf{f}_{\mathbf{X}}(\mathbf{x}) & \text { Mean } & \text { Variance } \\ \text { Gaussian } & \frac{1}{\sqrt{2 \pi \sigma^{2}}} \mathrm{e}^{-(\mathrm{x}-\mu)^{2} / 2 \sigma^{2}} & \mu & \sigma^{2} \\ \text { Exponential } & c e^{-\mathrm{cx}} & \frac{1}{\mathrm{c}} & \frac{1}{\mathrm{c}^{2}} \\ \quad \mathrm{E}(\mathrm{c}) & \mathrm{x} \geq 0, \mathrm{c}>0 & & \\ \text { Binomial } & \left(\begin{array}{c}\mathrm{n} \\ \mathrm{L}\end{array}\right) \mathrm{p}^{\mathrm{L}} \mathrm{q}^{\mathrm{n}-\mathrm{L}} & \mathrm{n} \mathrm{p} & \mathrm{n} \mathrm{p} \mathrm{L} \\ \quad \mathrm{B}(\mathrm{n}, \mathrm{p}) & \mathrm{L}=0, \ldots n ; p+q=1 & \end{array}$

Where $L$ is the number of looks to establish the probability of ID and $f_{\mathrm{x}}(x)$ is the probability density function. To determine the probabilities associated together in the ACT*IVE system, we have to normalize the values to determine the weighted probabilities. We can utilize a Kalman filter to update the state based on measurement information compared to the predicted reliability state.

$$
\left[\begin{array}{c}
\mathrm{A}_{\mathrm{k}+1} \\
\mathrm{C}_{\mathrm{k}+1} \\
\mathrm{~T}_{\mathrm{k}+1}
\end{array}\right]=\left[\begin{array}{lll}
\mathrm{p}_{\mathrm{ia}} & \mathrm{p}_{\mathrm{ic}} & \mathrm{p}_{\mathrm{it}} \\
\mathrm{p}_{\mathrm{va}} & \mathrm{p}_{\mathrm{vc}} & \mathrm{p}_{\mathrm{vt}} \\
\mathrm{p}_{\mathrm{ea}} & \mathrm{p}_{\mathrm{ec}} & \mathrm{p}_{\mathrm{et}}
\end{array}\right]\left[\begin{array}{c}
\mathrm{A}_{\mathrm{k}} \\
\mathrm{C}_{\mathrm{k}} \\
\mathrm{T}_{\mathrm{k}}
\end{array}\right]+\left[\begin{array}{c}
\mathrm{v}_{\mathrm{k}} \\
\mathrm{w}_{\mathrm{k}} \\
\mathrm{z}_{\mathrm{k}}
\end{array}\right]
$$

where, $\mathrm{v}_{\mathrm{k}}=\sigma^{2}, \mathrm{w}_{\mathrm{k}}=1 / \mathrm{c}^{2}$, and $\mathrm{z}_{\mathrm{k}}=\mathrm{n} \mathrm{p} \mathrm{L}$.

\section{Problem Description}

We seek to have a metric of reliability to determine whether or not the system is utilizing the fusion information in a proper way. Since the goal of the system is to make accurate and timely decisions, we need to address whether or not the fusion-user system is making the correct decision. A threshold can be set from the fusion system as to the quality of the input data; however, the combined analysis needs to stress the importance of the data. If a high confident accurate value is late, it still may be of use, just as a timely some-what confident data is.

In a tracking scenario, if the data is coming fast and slightly accurate, the human can see the pattern which is happening and correct for the positional errors. However, if the update rate is slow, then a high-confident positional information is desired. The problem is to address the timeliness of measurements in a track and ID system. For the scenario of interest, we have sensors that can track the two targets and the variability of update rates is undetermined. For the track system, we use the belief filter with state and measurement equations for all targets as:

$$
\begin{aligned}
& \hat{x}(k+1 \mid k)=F(x) \hat{x}(k \mid k) \\
& \hat{z}(k+1 \mid k)=H(k+1) \hat{x}(k+1 \mid k)
\end{aligned}
$$

with no bias (slowly time varying) error. For the scenario, we are interested in the update rate. If we have a highupdate and low accuracy, we get Figure 7.

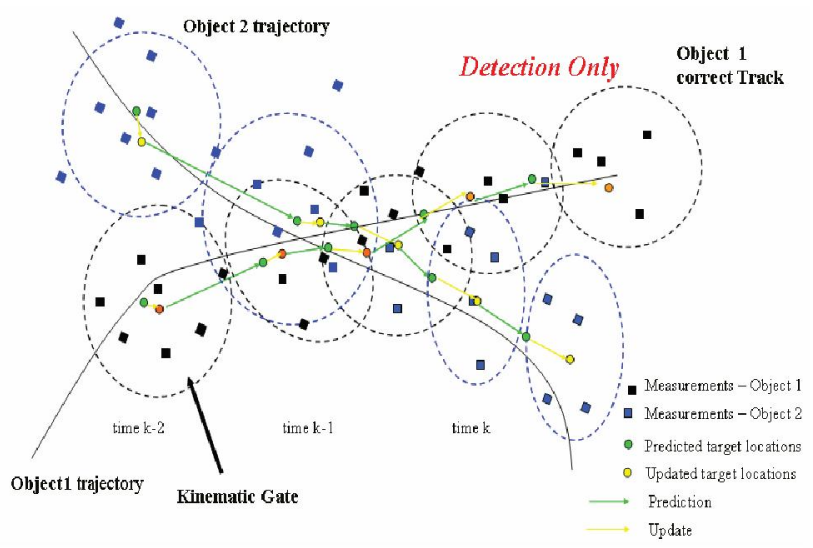

Figure 7. High Update Low Accuracy

and with the chance of timely confident classification information, we get Figure 8.

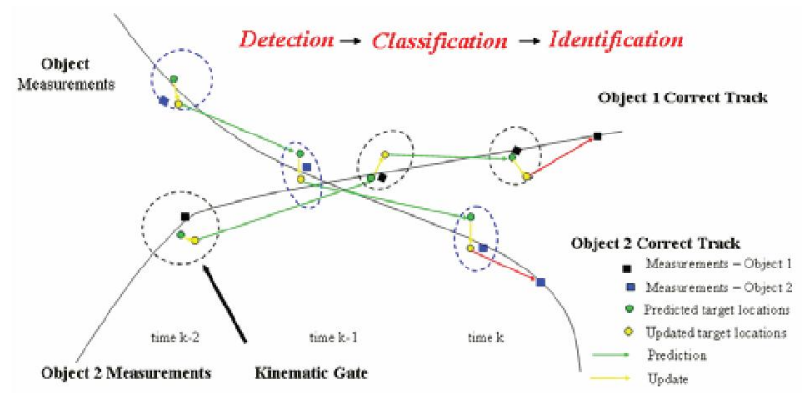

Figure 8. Low Update High Accuracy

Where $\mathrm{P}_{\mathrm{FA}}$ is Poisson distribution, mean number of FAs per frame, and $P_{D}$ depends on signal/noise ratio which varies with target range as $1 / r^{4}$ (active) or $1 / r^{2}$ (passive) [specify range $r_{0}$ for $\mathrm{P}_{\mathrm{D}}=0.8$ ].

\section{Results}

Figure 9 shows a two-track system with ID confidence 
and positional accuracy. The results we run using an exponential timeliness variance to allow the user strategy to select the expected combination of reliability metrics.
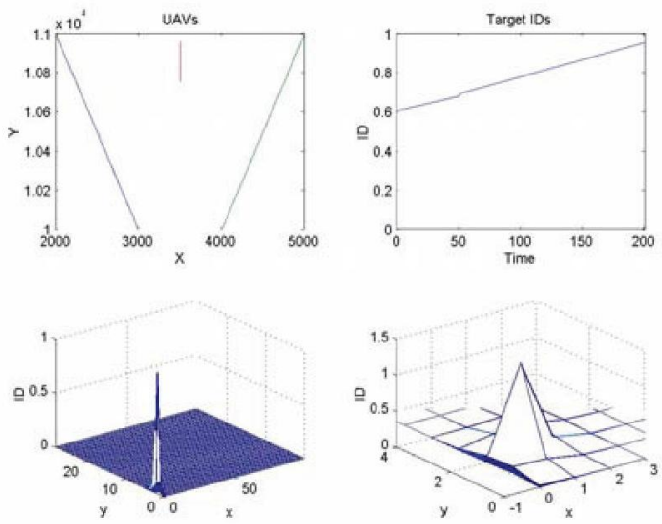

Figure 9. Selection of timeliness results.

Figure 10 shows that the appropriate selection of the user utility weights results in a quality fusion gain from selecting over the desirable reliability information.

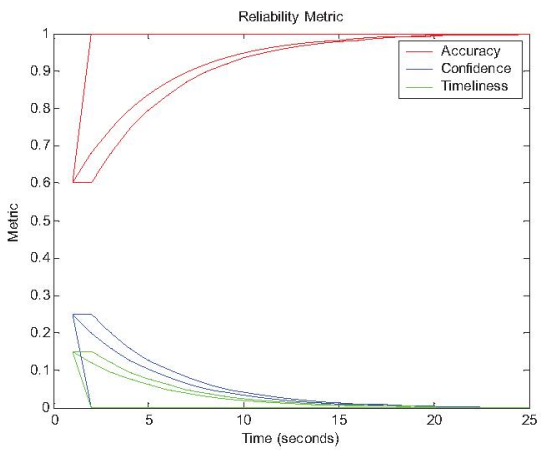

Figure 10. Selection of metrics.

Figure 11 is an estimated result from the system that tries to summarize the interaction between reliability and utility.

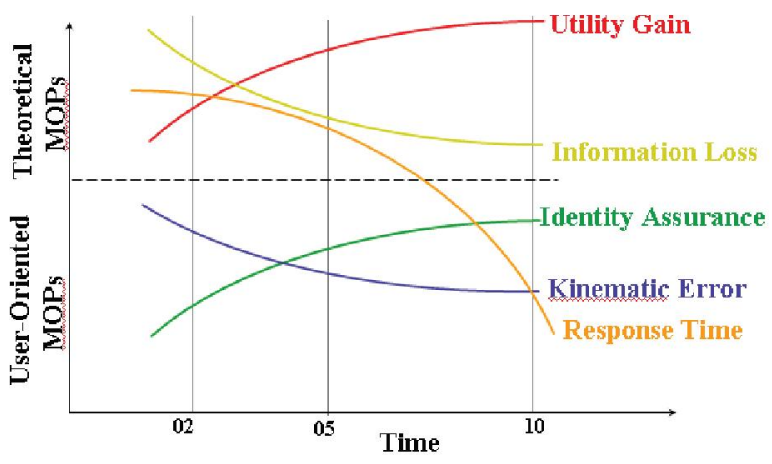

Figure 11. Utility Gain of the System.

\section{Conclusions}

We have presented a fusion reliability metric that combines accuracy, confidence, and timeliness. Typically, the user reliability information is not address in fusion systems and the analysis allows the user to refine the fusion process based on reliability constraints. The results show that the user selected the maximum utility based on the revisit rate of the sensors over the target in the search area based on a performance model of the prediction of the sensor reliabilities.

\section{References}

[1] E. Waltz and J. Llinas, Multi-Sensor Data Fusion, Artech House, Norwood, MA, 1990.

[2] E. Blasch and S. Plano, "JDL Level 5 Fusion model "user refinement' issues and applications in group tracking", SPIE Aerosense 4729, April 2002.

[3] $\mathrm{N}$ Xiong, and $\mathrm{P}$ Svensson, "Multisensor Management for information fusion: issues and approaches", Information Fusion, 3 , 2002, 163-186.

[4] Y. Zang, Q. Ji, and C. Looney, "Active Information Fusion for Decision Making under uncertainty," Fusion02, 2002.

[5] A. Worm, "Tactical Mission Analysis by means of Naturalistic Decision Making and Cognitive Systems Engineering," 1998.

[6] E. Blasch "Assembling an Information-fused Human-Computer Cognitive Decision Making Tool," IEEE AES Magazine, June 2000, pp. 11-17.

[7] M. R. Endsley, "Predictive utility of an objective measure of Situation awareness." In Pro of the HFS 34th Ann Mig. Santa Monica, CA: HFES, 1990.

[8] M. Rabin, "Risk Aversion and Expected-Utility Theory: A Calibration Theorem," Institute of Business and Economic Research, 2000.

[9] A. Motro, P. Anokhin, and A. C. Acar, "Utility-based resolution of Data Inconsistencies", 2003, ISE-TR-03-07, George Mason Univ.

[10] S. Andriole \& L. Adelman, Cognitive Systems Eng. for UserComputer Interface Design, Prototyping, \& Evaluation, Lawrence Erlbaum, 1995.

[11] B. Fischhoff, "Hindsight does not equal foresight: The effect of outcome knowledge on judgment under uncertainty." Journal of Experimental Psychology: Human Performance \& Perception, 1975, 1, 288-299.

[12] H. Simon, Models of Bounded Rationality, Vols. 1 and 2. MIT Press, 1982.

[13] S. Blackman, Multiple-Target Tracking with Radar Applications, Artech House, Norwood MA, 1986.

[14] J. Reason, Human Error, Cambridge University Press, 1990

[15] D. Hall, Mathematical Techniques in Multisensor Fusion, Artech House, Norwood, MA, 1992.

[16] K. Tversky and D. Kahneman, Judgment Under Uncertainty, Utility, Probability, \& Decision Making, Wendt and Vlek (Eds,), D. Reidel Pub, Boston, 1973.

[17] J. Nash, "Optimal Allocation of Tracking Resources," Proc. IEEE CDC, 1977, pp. 1177-1180

[18] Fishburn, P. C., Nonlinear Preference and Utility Theory, The Johns Hopkins University Press, Baltimore, Maryland, 1988.

[19] E. Blasch, Derivation of A Belief Filter for High Range Resolution Radar Simultaneous Target Tracking and Identification, Ph.D. Dissertation, Wright State University, 1999.

[20] A. Papoulis \& S Pillai, Probability and Random Variables and Stochastic Processes, McGraw Hill, 2001.

[21] P. M. Fitts. "The information capacity of the human motor system in controlling the amplitude of movement." J. Exp. Psychology, Vol. 47, No. 6, 1954, pp. 381-391. 